\title{
Cirurgia de implantes guiada por computador: relato de caso clínico
}

\section{Computer-guided implant surgery: clinical case report}

\section{Thomas Altmann Moraes de Macedo', Ana Cybele Queiroz Cunha², Jefferson David Melo de Matos $^{3}$, Antonio Walter Brito Neto ${ }^{4}$, Victor Archeti Vardiero ${ }^{5}$, Tiago França Araripe Cariri ${ }^{6}$}

'Autor para correspondência. Centro Universitário Doutor Leão Sampaio (UNILEÃO). Juazeiro do Norte, Ceará, Brasil. thomas-macedo@hotmail.com ${ }^{2}$ Centro Universitário Doutor Leão Sampaio (UNILEÃO). Juazeiro do Norte, Ceará, Brasil. anacybeleqc@gmail.com ${ }^{3}$ Departamento de Prótese Dentária, Universidade Estadual Paulista Júlio de Mesquita Filho UNESP. São José dos Campos. São Paulo, Brasil, jefferson.matos@ict.unesp.br ${ }^{4}$ Departamento de Odontologia, San Leopoldo Mandic. Campinas, São Paulo, Brasil. drwalter_neto@hotmail.com ${ }^{5}$ Departamento de Odontologia, Centro Caririense de Pós-Graduação (CECAP). Juazeiro do Norte, Ceará, Brasil. victorvardiero@yahoo.com.br ${ }^{6}$ Departamento de Odontologia, Centro Universitário Doutor Leão Sampaio (UNILEÃO). Juazeiro do Norte, Ceará, Brasil. dr.tiagofranca@hotmail.com

RESUMO I Este estudo tem por objetivo descrever as etapas do planejamento, a funcionalidade da cirurgia de implantes guiada por computador, suas aplicações clínicas, bem como relatar um caso clínico, a cerca desse tipo de procedimento realizado em um serviço público no Brasil. Paciente F.D.C.O., gênero masculino, melanoderma, 54 anos de idade, compareceu ao Setor de Prótese Dentária do Centro de Especialidades Odontológicas Regional (CEO-R) do Crato - CE - Brasil, para reabilitação. Foi realizado confecção de próteses totais superior e inferior. Durante todo o tratamento no Setor, foi observada dificuldade de confecção da peça inferior e o paciente foi alertado que devido a reabsorção do rebordo nessa arcada, a retenção e a estabilidade provavelmente ficariam comprometidas. Diante do quadro, o mesmo foi encaminhado para uma avaliação do Setor de Cirurgia e Traumatologia Buco Maxilo Facial visando a possibilidade de enquadramento no programa de implantes dentários que se iniciava no centro de especialidades. Desde o surgimento da osseointegração os implantes dentários tornaram-se uma solução viável para o tratamento de paciente desdentados totais ou parciais. A cirurgia virtual guiada é uma opção que deve melhorar a precisão, pois proporciona um bom conhecimento anatômico da área a ser reabilitada, aprimorando então a qualidade da técnica cirúrgica, que quando associada ao desenho do implante, promove uma estabilidade primária apropriada. A cirurgia guiada de instalação dos implantes é realizada sem incisão, com as perfurações sendo feitas através do tecido mole, permitindo a precisa confecção dos alvéolos cirúrgicos artificiais e a instalação dos implantes. Quando existe a possibilidade da realização deste procedimento, o resultado é de mínimo trauma cirúrgico e excelente pós-operatório para o paciente.

PALAVRAS-CHAVE: Implantes Dentários, Reabilitação Bucal, Tomografia Computadorizada.
ABSTRACT | This study aims to describe the planning stages, the functionality of this technique, its clinical applications, as well as to report the first case of this type of procedure performed in a public service in Brazil. Patient F.D.C.O., male, melanoderma, 54 years old, attended the Dental Rehabilitation Sector of the Regional Dental Specialties Center (CEO-R) of Crato - CE, for rehabilitation. The upper and lower total prostheses were made. During the entire treatment in the Sector, it was observed the difficulty of making the lower part and alerted to the patient that due to reabsorption of the ridge in the arch, retention and stability would probably be compromised. In front of the table, the same was sent to an evaluation of the Oral Maxillofacial Surgery and Traumatology Sector aiming at the possibility of framing the dental implant program that began at the specialty center. Since the onset of osseointegration, dental implants have become a viable solution for the treatment of total or partial edentulous patients. In the last decades, the use of osseointegratable implants in edentulous patients has innovated the functional rehabilitation, since the treatment has a longevity and a clinical effectiveness proven by several studies. Guided virtual surgery is excellent when it comes to precision, since it provides us with a good anatomical knowledge of the area to be rehabilitated, thus improving the quality of the surgical technique, which, when included in the implant design, promotes an appropriate primary stability. Guided implant implant surgery is performed without incision, with perforations being made through the soft tissue, allowing the precise preparation of the artificial surgical alveoli and the installation of the implants. When it is possible to perform this procedure, the result is minimal surgical trauma and excellent postoperative for the patient.

KEYWORD: Dental Implants; Oral Rehabilitation; Computed Tomography. 


\section{Introdução}

grande desafio da Odontologia contemporânea, tem sido de reestabelecer ao paciente invalido oral; a função, fonética, conforto e saúde do sistema estomatognático. Na Odontologia tradicional, era visto que quanto mais edêntulo fosse o paciente, maior seria a dificuldade em planejar e reabilitar o caso. A Implantodontia, com o advento da osseointegração, veio suprir essa lacuna e, desse modo, tornou-se possível o desenvolvimento de uma nova filosofia de reabilitação com implantes: a cirurgia virtual guiada'.

A osseointegração é definida como uma conexão direta estrutural e funcional entre o osso vivo remodelado, e a superfície de um implante submetido a carga funcional; a criação e manutenção da osseointegração depende da capacidade de cicatrização, reparação e remodelação dos tecidos. Um implante osseointegrado é similar a um dente com anquilose não reabsorvido, dessa forma a resposta óssea aos implantes deste tipo é positiva e a ancoragem entre osso e implante osseointegrados é firme, garantindo o sucesso da instalação².

O sucesso da instalação dos implantes, bem como a posição do implante estão intimamente relacionados com osseointegração, dessa forma o planejamento prévio é necessário para que se possa alcançar com êxito a reabilitação, devolvendo forma e função na qual foi perdida ${ }^{3}$. Os casos que eram reabilitados com implantes osseointegraveis tinham como primeiro passo a realização de exames radiográficos convencionais, onde estes, influenciavam no comprimento dos implantes, considerando a altura óssea disponível como principal ponto. No entanto, decisões importantes como a posição e a inclinação eram definidas apenas durante $o$ ato cirúrgico, por esta razão ocasionavam falhas estéticas e funcionais ${ }^{4}$.

Com os avanços das técnicas imagenológicas, permitiu-se um grande salto para a Implantodontia, auxiliando no planejamento e na execução das reabilitações orais ${ }^{5}$. As tomografias computadorizadas e as ressonâncias magnéticas dispoem imagens de alta qualidade que permitem uma análise detalhada da condição óssea e das áreas anatômicas a serem utilizadas, dessa forma a criação de um protótipo (guia) 3D, tornou as cirurgias de implantes menos invasivas e com procedimentos cirúrgicos mais precisos, isso nos casos em que o planejamento é efetuado corretamente ${ }^{6}$.

Diversos trabalhos relatam o sucesso desta técnica, todavia, a utilização da cirurgia guiada, ainda é pouco descrita na literatura, desta forma, este trabalho tem por objetivo descrever as etapas do planejamento, a funcionalidade desta técnica e suas aplicações clínicas.

\section{Relato de caso}

Paciente F.D.C.O., gênero masculino, melanoderma, 54 anos de idade, compareceu ao Setor de Prótese Dentária do Centro de Especialidades Odontológicas Regional (CEO-R) do Crato - CE - Brasil, para reabilitação. O paciente apresentava como queixa principal um aumento de volume na região anterior da maxila; foi realizado confecção de próteses totais superior e inferior. Durante todo o tratamento no Setor, foi observada a dificuldade de confecção da peça inferior e alertado para o paciente que devido à reabsorção do rebordo nessa arcada (FIG. 1), a retenção e estabilidade provavelmente ficariam comprometidas.

Figura 1. Pré-operatório, observando rebordo alveolar em lâmina de faca, com grande reabsorção.

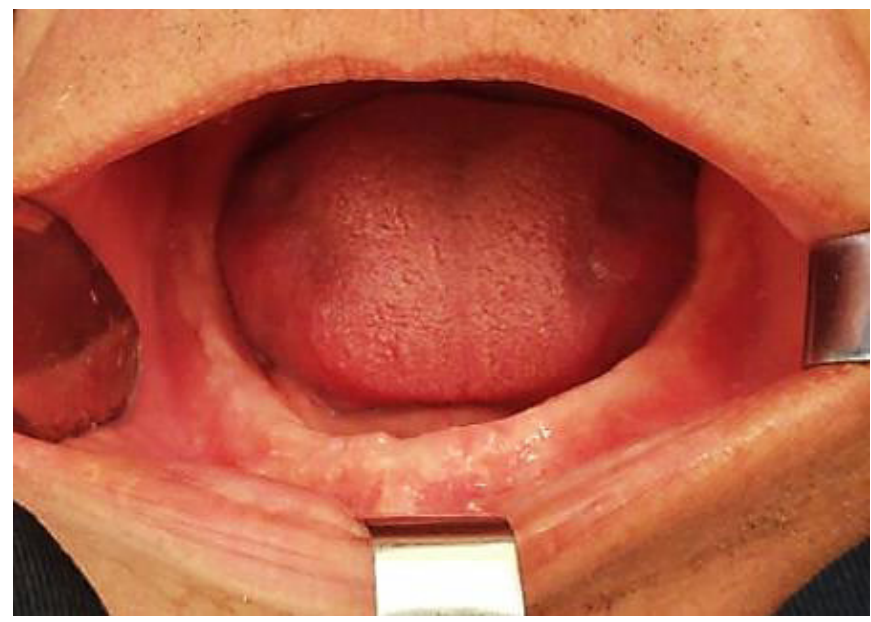


Ao término do tratamento, a suspeita foi confirmada e o paciente não conseguiu adaptação mínima e não fazia o uso da prótese inferior. Diante do quadro, o mesmo foi encaminhado para uma avaliação do Setor de Cirurgia e Traumatologia Buco Maxilo Facial visando a possibilidade de enquadramento no programa de implantes dentários que se iniciava no centro de especialidades.

Paciente normosistemico, não relatou nenhum tratamento médico ou uso de nenhuma medicação, bem como não havia histórico de crise alérgica. Após avaliação inicial, verificou-se que ele se enquadrava nos parâmetros clínicos e socioeconômicos pré-estabelecidos pelo serviço de Implantodontia. O tratamento proposto para o caso, foi explicado de forma clara e suscinta, em seguida logo o mesmo aceitou.

Foi realizado um planejamento cirúrgico, minimamente invasivo, com a utilização de guia, para instalação de cinco implantes, onde 3 implantes foram fixados entre os forames mentonianos e 2 após os forames (Figuras. $2 ; 3 ; 4 ; 5 ; 6$ ) para posterior instalação de prótese fixa (protocolo).

Figura 2. TC (com visão panorâmica) mostrando o posicionamento no planejamento dos implantes.

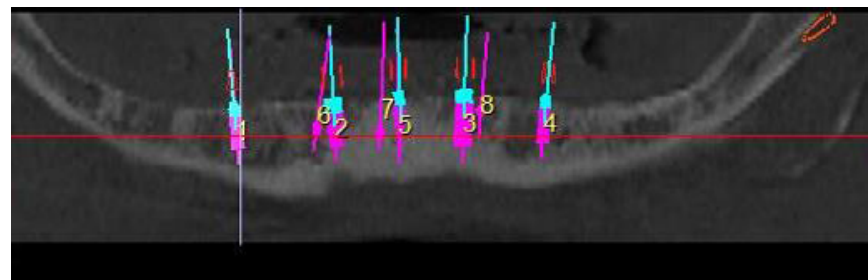

Figura 3. Reconstrução 3D da arcada inferior, observando adaptação da prótese e planejamento.

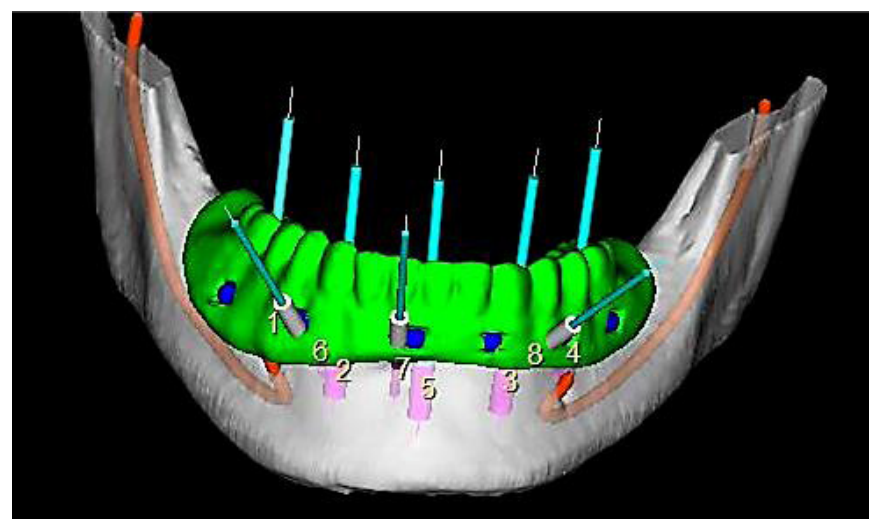

Figura 4. Corte axial evidenciando a distribuição dos implantes

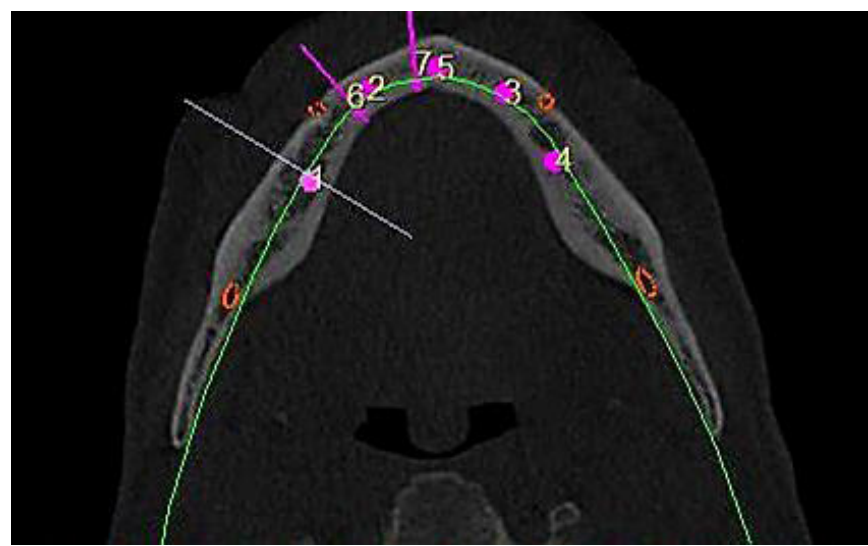

Figura 5. Reconstrução 3D da arcada inferior, observando o posicionamento dos implantes.

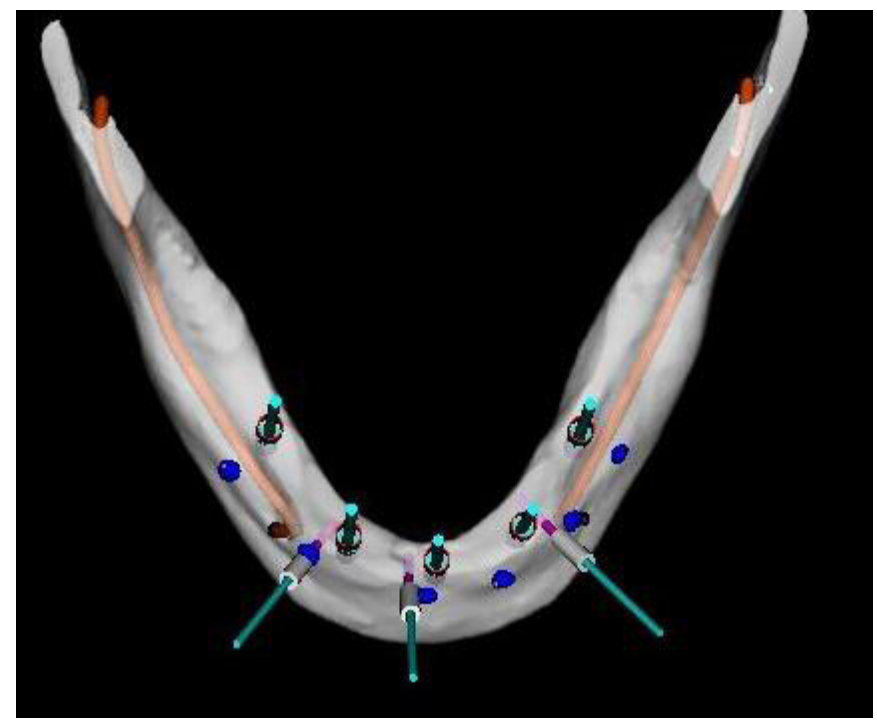

Figura 6. Reconstrução 3D da arcada inferior, observando a relação dos implantes com o forame mentoniano.

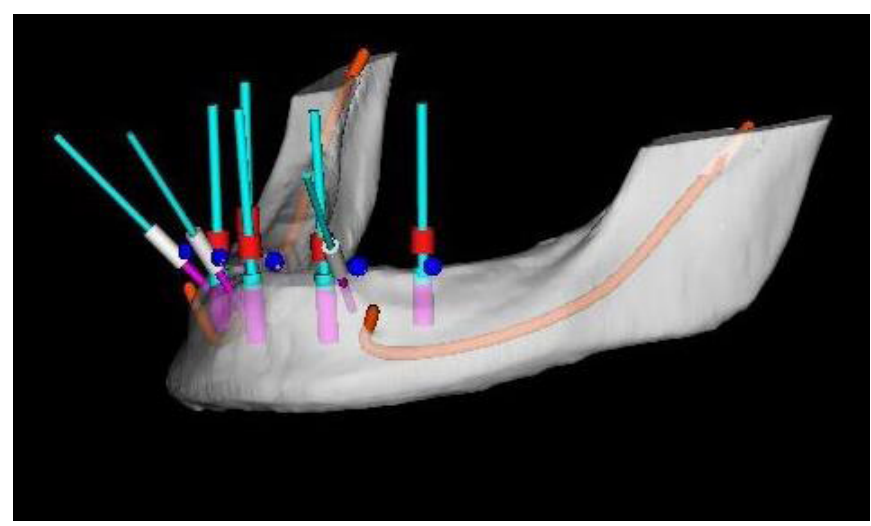


Para a confecção do guia cirúrgico, a prótese inferior sem estabilidade, realizada pelo Setor específico do CEO - $R$ foi utilizada, visto que os dentes desta prótese estavam montados em posição satisfatória e assim poderiam servir de referência protética. Inicialmente foram realizadas marcações, usando uma broca esférica de 2,0 mm na flange, sem ultrapassa-la (FIG. 7). Esses orifícios foram preenchidos com guta percha e serviram de referencia para o software DentalSlice - Bioparts (FIG. 8). O paciente realizou a tomografia computadorizada utilizando a prótese, em oclusão, permitindo que a mesma, permanecesse sem sofrer movimentações durante todo o exame. No final, obteve-se uma imagem tomográfica que pôde ser estudada em todos os 3 cortes de orientação, com a prótese posicionada no rebordo alveolar. Essa imagem serviu de planejamento dos implantes, bem como, possibilitar a prototipagem do guia (FIG. 09) com uma correta adaptação ao paciente. $O$ guia cirúrgico foi feito pela bioparts para 4 implantes ou mais - RAPTOR Bioparts (Cód: 44 /T-248).

Figura 7. Marcação do guia tomográfico

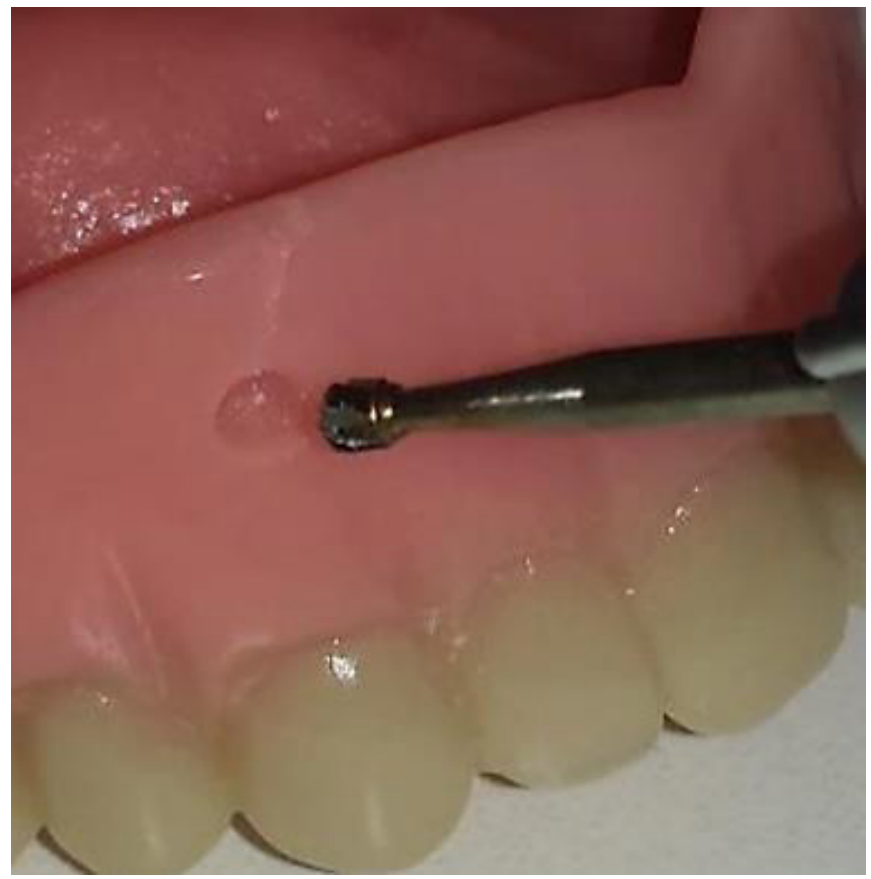

Fonte: www.bioparts.com.br/notastecnicas
Figura 8. Orificios preenchidos com guta percha

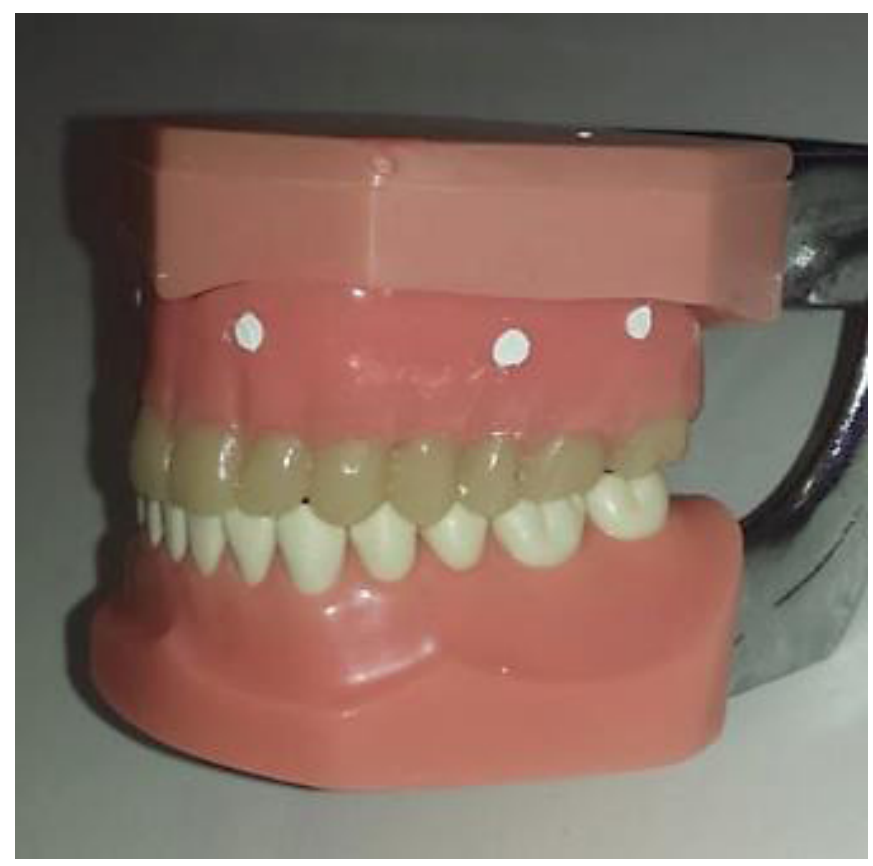

Fonte: www.bioparts.com.br/notastecnicas

Figura 9. Guia cirúrgico prototipado.

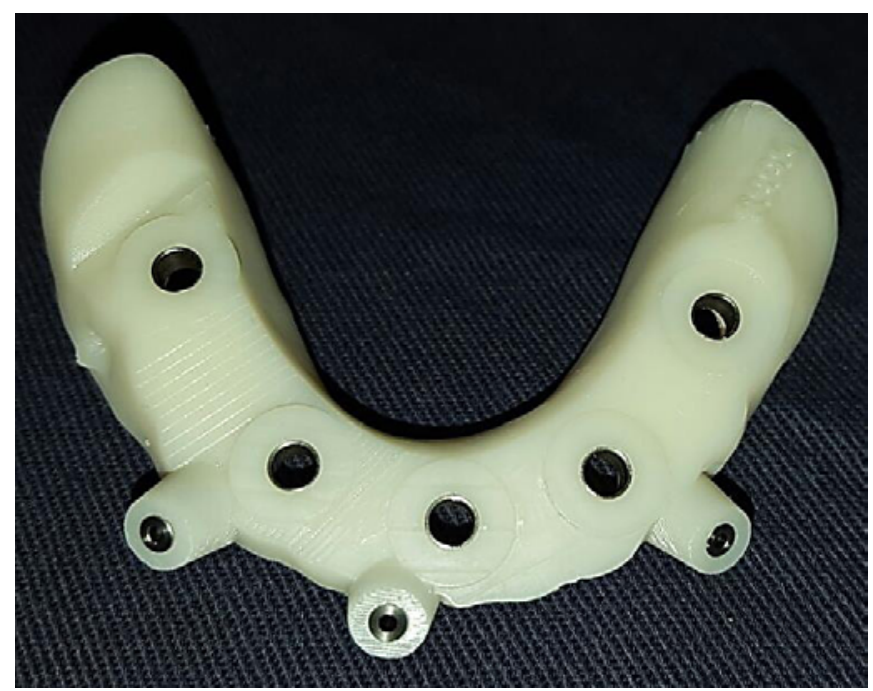

O procedimento cirúrgico foi realizado sob anestesia local, utilizando-se 3 tubetes de articaína a $4 \%$, pois a mesma dispõe de um rápido início de ação e sua principal indicação é para anestésia infiltrativa com uma duração intermediária. Inicialmente realizou-se a antissepsia extra e intra oral, bem como a 
aposição de campos cirúrgicos; o guia cirúrgico foi desisfectado com clorexidina a 2,0\%, durante trinta minutos, estabilizado e fixado na mandíbula do paciente (FIG. 10). Foram realizadas as devidas medicações pré-operatórias, com amoxicilina $875 \mathrm{mg}$ um dia antes do procedimento cirúrgico, no espaço de 12 em 12 horas, durante o período de 7 dias; ainda foi orientado quanto a ingestão de uma medicação pós-operatória de nimesulida $200 \mathrm{mg}$ no decorrer de 24 horas, durante três dias e dipirona sódica $500 \mathrm{mg}$, um comprimido a cada seis horas, durante 24 horas. As perfurações foram realizadas respeitando a sequencia de brocas e altura e espessura em cada região.

Figura 10. Guia cirúrgico fixado.

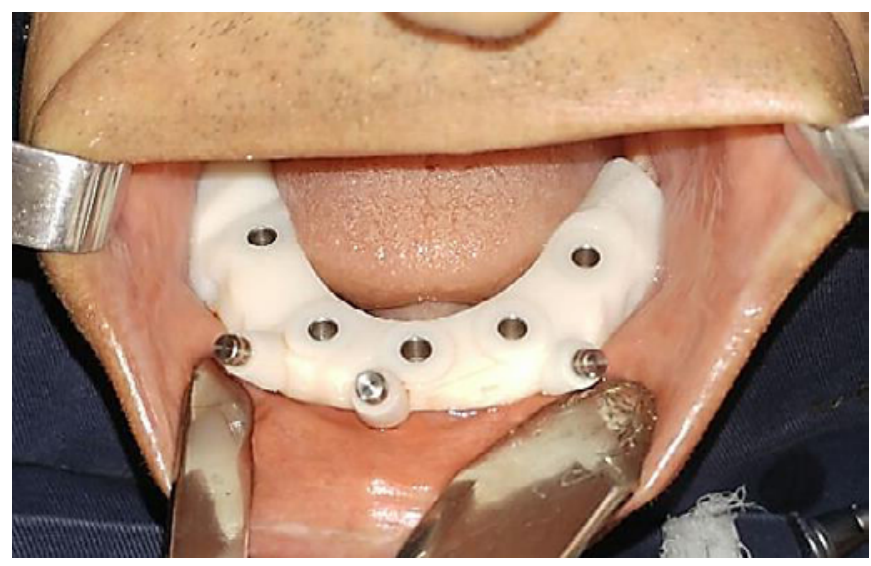

O kit de fixação do guia cirúrgico utilizado foi o Raptor da empresa Bioparts Prototipagem Biomédica (Brasília, Distrito Federal - Brasil). Esse kit possui uma broca de fixação específica e vários pinos de fixações do mesmo tamanho da broca (FIG. 11). A perfuração deverá ser realizada até o "stop" da broca (FIG. 12 A; B). Esse comprimento de perfuração é definido por uma anilha no guia de acordo com o planejamento tomográfico antes da confecção do mesmo, assim não importa quão profunda a fixação foi planejada, a anilha sempre compensará a distancia exata para a broca e para $\circ$ pino de fixação durante a cirurgia.
Figura 11. Kit de fixação do guia cirúrgico.

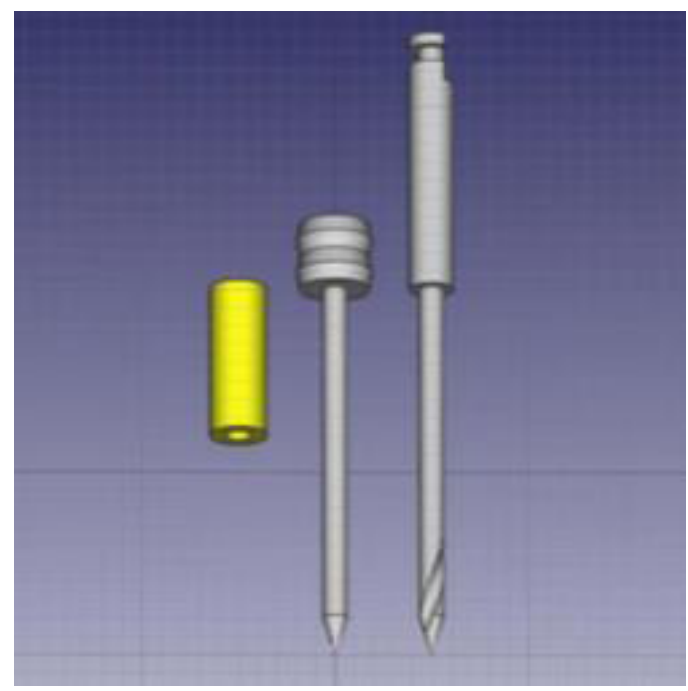

Fonte: www.bioparts.com.br/notastecnicas

Figura 12. (A) Simulação da perfuração do guia ate o "stop". (B) Pino de fixação instalado
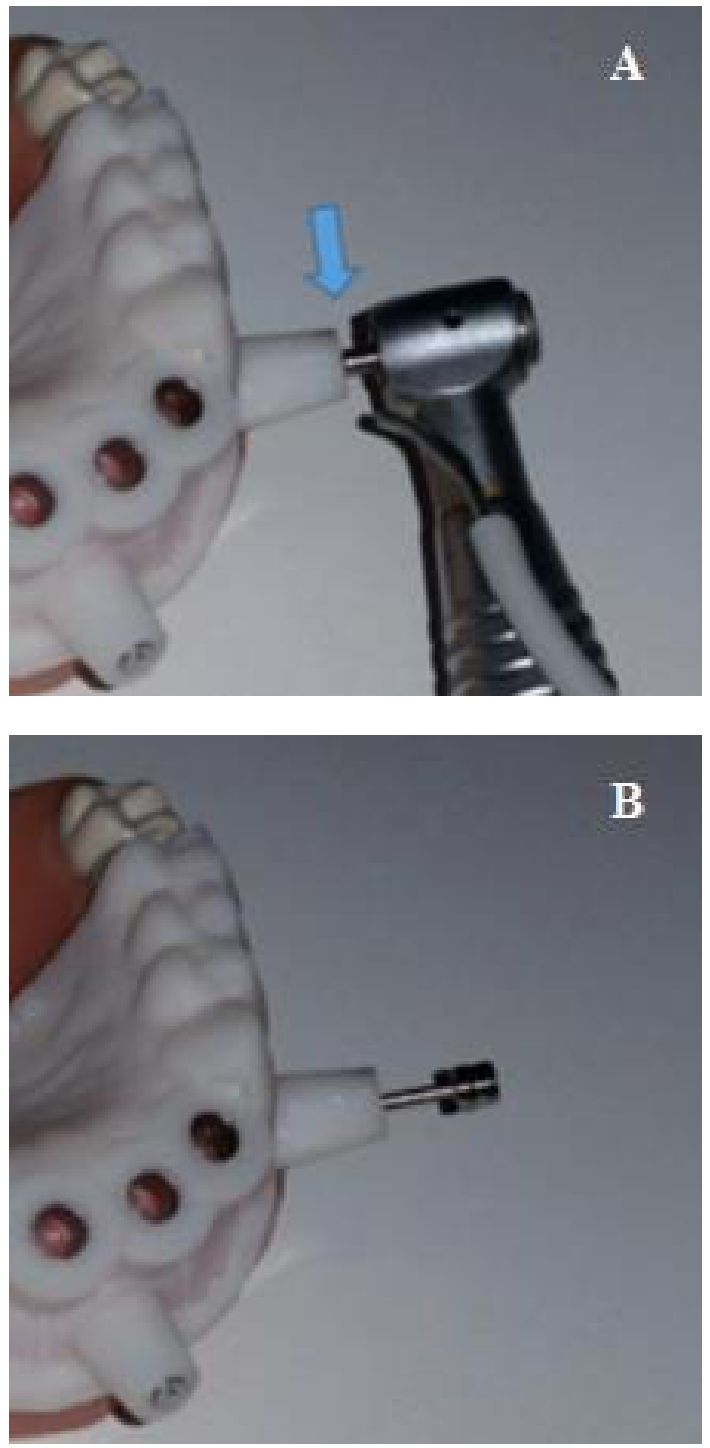

Fonte: www.bioparts.com.br/notastecnicas 
Durante a perfuração foi utilizado um guia de brocas (FIG. 13 A; B) do Kit Raptor para dar estabilidade e orientação as brocas; foi usado uma broca lança até romper a cortical óssea, em seguida utilizou da broca 2.0 até a mensuração de $7,0 \mathrm{~mm}$; ainda utilizou-se da broca 2.7 sobre a mesma milimetragem, logo após foi instalado dois implantes Universal III - Due Core, com $\varnothing 3.5 \times 7.0 \mathrm{~mm}$, isso na região posterior. Já na região anterior foi feita a mesma sequencia de brocas, no entando com uma perfuração de $9.0 \mathrm{~mm}$, com a seguida intalação de três implantes com $\varnothing 3.5 \times 9,0 \mathrm{~mm}$.

Figura 13. (A) Adaptação do guia de brocas do kit Raptor. (B) Torquímetro
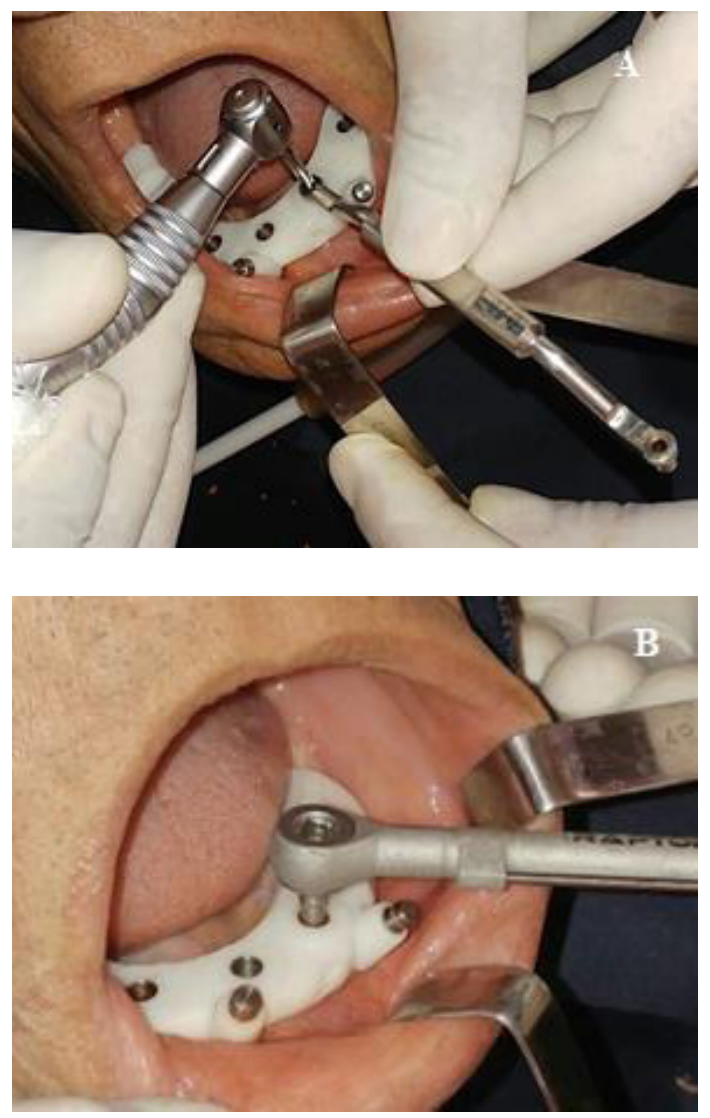

Os implantes foram instalados (FIG. 14), não havendo necessidade de incisões e/ou suturas, levando um tempo cirúrgico total de 40 minutos; orientou-se ainda $\circ$ próprio que alocasse compressas de gelo sobre a região, atuando de forma significativa no edema; o gelo por sua vez age como um anti-inflamatório natural. Todavia orientou-se, que o mesmo fizesse o uso de um analgésico e de um antiflamatório por três dias, logo após retornando ao consultório para observação da condição do caso.

\section{Discussão}

A procura por trazer uma maior segurança e comodidade para $\circ$ paciente durante uma cirurgia implantar tem sido apresentada em vários estudos ${ }^{7-10}$. A cirurgia guiada está relacionada diretamente nesse quesito, por oferecer maior precisão, uma vez que faz o uso de exames prévios; como a tomografia computadorizada que permitiu a reprodução dos tecidos mineralizados e áreas anatômicas com uma menor distorção e uma dose radiográfica mínima, possibilitando planejar virtualmente o guia cirúrgico, bem como o procedimento cirúrgico sem retalho, permitindo a recuperação rápida do paciente, livre de incômodos ${ }^{11-14}$. A importância da precisão cirúrgica, neste caso clínico, não foi só para o posicionamento ideal dos implantes, mas também pela proximidade com estruturas nobres, como o nervo alveolar inferior. $O$ planejamento prévio e a confecção do guia cirúrgico possibilitou uma maior precisão durante a instalação dos implantes, trazendo maior comodidade ao paciente ${ }^{15}$.

Quando bem indicada, a cirurgia guiada, fornece uma variedade de vantagens quando comparada aos procedimentos cirúrgicos convencionais, por ser menos invasiva, resulta em menos morbidade, menos dor pós-operatória, menos edema e um menor tempo de cicatrização. Os sintomas pós operatórios, neste caso, praticamente foram ausentes, não observado-se edema, o paciente relatou dor leve durante o primeiro dia, reduzindo o uso de analgésicos nos dias subsequentes ${ }^{16}$.

Durante o procedimento cirúrgico, é de suma importância que o guia prototipado mantenha-se justaposto ao rebordo, impossibilitando alterações de posição durante a sua fixaçãa ${ }^{17}$. A principal causa de falhas no protocolo da cirurgia virtual guiada está relacionada a esta etapa clínica; outro benefício da utilização desta técnica cirúrgica é a possibilidade da utilização de anilhas, para guiar na instalação dos implantes, com tamanhos e formas diferentes, tornando ainda maior a precisão da perfuração dos implantes. Dessa forma, leva-se em consideração as vantagens e as limitações, este procedimento é indicado como uma opção viável para reabilitação de mandíbulas e maxilas edêntulas, como foi demonstrado no caso clínico apresentado ${ }^{18}$. 
Algumas das desvantangens dessa técnica é o custo relativamente alto e pode ocorrer possíveis erros na confecção do guia, caso a tomografia possa vir com falhas; vale a pena elucidar que uma das principais limitações da técnica é possíveis discrepâncias na imagem tomográfica, isso atrapalhando de forma direta o tratamento proposto ao caso.

O guia cirúrgico é a chave desse novo método, visto que ele permite a transferência do planejamento virtual predeterminado para o planejamento real. Após escolher a posição dos implantes, a prótese virtual pode ser sobreposta à imagem para analisar a posição das fixações em relação à reabilitação, isso permitindo modificações e ajustes para definir uma posição ideal para os implantes ${ }^{19}$.

Uma nova técnica foi introduzida para o uso de guias cirúrgicos, visto que a instalação de implantes em pacientes edêntulos é de difícil manuseio, pois apresentam estabilidade insatisfatória durante 0 procedimento operatório, nesse contexto 0 uso de implantes transitórios para conferir dureza do guia no transoperatório é fundamental, ao posso que, após instalação dos implantes finais, os transitórios serão removidos. Os implantes transitórios utilizados neste caso foram fornecidos pelo kit de fixação Raport da empresa Bioparts, o que conferiu estabilidade do guia durante a perfuração dos alvéolos cirúrgicos ${ }^{20}$.

A cirurgia flapless demonstra algumas vantagens em relação ao protocolo tradicional em amostra realizadas em animais, onde os implantes instalados pelo método tradicional sofrem uma redução da estabilidade inicial nas primeiras 4 semanas, tendo sua estabilidade acrescida na quinta semana e 0 pico de estabilidade na 8 semana; já os implantes flapless não proporcionaram estabilidade semeIhante aos tradicionais nas 2 primeiras semanas, porém a estabilidade aumentou nas semanas seguintes e alcançou o pico em 8 semanas $^{21}$. Todavia, existe limitações na utilização desta técnica, seja um grau de reabsorção óssea alveolar e à presença de infecção aguda, além disso um nível adequado ao osso e aos tecidos moles que deverão instalar o implante na posição ideal em relação ao planejado e estabilização do modelo cirúrgico.
Com o surgimento das tomografias computadorizadas, com suas secções coronais e axiais, a observação das estruturas anatômicas é mais precisa e nítida. Essas imagens (proporções 1:1), são introduzidas em softwares específicos de manipulação de dados, onde podem ser manuseadas possibilitando uma simulação virtual na instalação de implantes. Todo o planejamento virtual é fornecido ao paciente através do guia cirúrgico, que é confeccionado a partir da cirurgia virtual ${ }^{22}$. Este guia nos orienta quanto à posição das perfurações ideais, à medida que o software permite o planejamento da posição ideal dos implantes e o guia imprime este posicionamento com precisão para $\circ$ paciente, tornando o procedimento cirúrgico mais rápido, com menor trauma as tecidos orais e menor chances de dor pós-operatória ${ }^{23}$.

A utilização de um software que simule o procedimento cirúrgico traz uma grande precisão e previsibilidade no tratamento; as cirurgias sem retalho são o padrão-ouro da implantodontia moderna, e a implementação de um software que permita o planejamento virtual torna esse tipo de intervenção muito mais segura e previsível ${ }^{24-26}$. Dessa forma, durante o planejamento deste relato de caso, foi possível observar a relação entre o osso e os futuros implantes em uma imagem tridimensional no pré-operatório, permitindo uma exata precisão na instalação dos implantes ${ }^{27}$.

Atualmente é observada uma nova era no que cerne ao planejamento cirúrgico, o que se tornou possível pelo avanço da área imagenológica, tornando os procedimentos mais rápidos e menos invasivos 28 . Um dos primeiros relatos de utilização de guias cirúrgicos criados em um computador foi feito por um estudo com oito pacientes, nos quais os guias cirúrgicos eram acomodados no rebordo alveolar após o descolamento do retalho; satisfeitos com os resultados obtidos neste estudo, os pesquisadores criaram a cirurgia sem retalho, tornando a cirurgia mais rápida e menos invasiva ${ }^{29-31}$. 


\section{Conclusão}

Pode concluir-se a partir deste estudo que:

A cirurgia virtual guiada apresenta uma alta precisão no posicionamento tridimensional dos implantes, pois esta é orientada pelo posicionamento virtual das estruturas anatômicas a serem reabilitadas. Dessa forma, consegue-se um resultado muito próximo do planejado, já que o próprio guia evita erros inerentes ao operador. Este procedimento ainda permite que se instale, com segurança, implantes com as maiores dimensões possíveis, o que facilita a estabilidade primária e permite que estes entrem em função precocemente.

\section{Contribuição dos autores}

Macedo TAM, participou da etapa de conceitos e ideias para a pesquisa em questão, confecção do projeto, definição do tema intelectual, busca bibliográfica, tabelação dos dados, análise dos dados, preparação, revisão e edição do manuscrito. Cunha $A C Q$, participou da etapa de conceitos e ideias para a pesquisa em questão, confecção do projeto, definição do tema intelectual, busca bibliográfica, tabelação dos dados, análise dos dados, preparação, revisão e edição do manuscrito. Matos JDM, contribuiu na fase de busca bibliográfica nas bases de catalogação bibliográfica. Neto AWB, participou na fase de preparação, revisão e edição do manuscrito. Vardiero VA, participou da etapa de conceitos e ideias para a pesquisa em questão, confecção do projeto, definição do tema intelectual, preparação, revisão e edição do manuscrito. Cariri TFA, participou da etapa de conceitos e ideias para a pesquisa em questão, confecção do projeto, definição do tema intelectual, preparação, revisão e edição do manuscrito.

\section{Conflitos de interesses}

Nenhum conflito financeiro, legal ou político envolvendo terceiros (governo, empresas e fundações privadas, etc.) foi declarado para nenhum aspecto do trabalho submetido (incluindo mas não limitandose a subvenções e financiamentos, conselho consultivo, desenho de estudo, preparação de manuscrito, análise estatística, etc).

\section{References}

1. Adell R, Lekholm U, Rockler B, Brånemark PI. A 15-year study of osseointegrated implants in the treatment of the edentulous jaw. Int J Oral Surg. 1981;10(6):387-416.

2. Albaricci M, Queiroz TP, Marcantonio Jr E, Margonar R. Planejamento virtual para a realização de cirurgia "flapless" e prótese imediata. Relato de caso clínico. Rev Odontol UNESP. 2010;39(especial):111.
3. Bernardes SR, Claudino M, Sartori IAM. Relevância clínica do tratamento de superfície de implantes dentários. Jornal ILAPEO. $2012 ; 6(2): 65-74$.

4. Bioparts Prototipagem Biomédica. Notas Técnicas [Internet]. 2015 [acesso em 2017 jun. 09]. Disponível em: http://bioparts.com.br/notas-tecnicas/

5. Branemark PI, Hansson BO, Adell R, Breine U, Lindstrom J, Hallén $O$ et al. Osseointegrated implants in the treatment of the edentulous jaw. Experience from a 10-year period. Scand J Plast Reconstr Surg Suppl. 1977;16:1-132.

6. Carvalho RS, Francischone Junior CE, Kobayashi FM, Scarafissi PFS, Costa APRM, Francischone CE. Novo implante P-I Brånemark PhilosophyTM e cirurgia guiada por computador: inovações tecnológicas inaugurando uma nova era na Implantologia. Rev Dental Press Periodontia Implantol. $2007 ; 1(3): 74-86$.

7. Carvalho NB, Gonçalves SLMB, Guerra CMF; Carreiro AFP. Planejamento em implantodontia: uma visão contemporânea. Rev Cir Traumatol Buco-Maxilo-Fac. 2006;6(4):17-22.

8. Casap N, Tarazi E, Wexler A, Sonnenfeld U, Lustmann J. Intraoperative computadorized navigation for flapless implant surgery and immediate loading in the edentulous mandible. Int J Oral Maxillofac Implants. 2005;20(1):92-8.

9. Cremonini CC, Feng HS, Nakazato A, Nagano CP, Batista OHP, Hayek RRA, Lima LA. Utilização de guias cirúrgicas para colocação de implantes dentários: revisão de literatura. Braz J Periodontol. 2015; 25(2):40-47.

10. Dinato JC, Nunes LS. Tratamento protético sobre implante no desdentado total na atualidade. Implant News. 2006;3(5):1 2-20.

11. Faverani LP, Ferreira GR, Gaetti-Jardim EC, Okamoto R, Shinohara EH, Assunção WG, Garcia Júnior IR. Implantes osseointegrados: evolução sucesso. Salusvita. 2011 ; 30(1):4758.

12. Geng W, Liu C, Su Y, Li J, Zhou Y. Accuracy of different types of computer-aided design/ computer-aided manufacturing surgical guides for dental implant placement. Int J Clin Exp Med. 2015;8(6):8442-8449.

13. Holcman M, Pinto JT, Farina CG, Falsi M, Santos Neto A, Vasconcelos AP et al. Cirurgia guiada em função imediata: proposta de técnica sem incisão. Rev Bras Implant. 2007;13(2):6-9.

14. Hultin $M$, Svensson KG, Trulsson M. Clinical advantages of computer-guided implant placement: a systematic review. Clin Oral Implants Res. 2012;23(6):124-135. doi: $10.1111 / \mathrm{i} .1600-0501.2012 .02545 . x$ 
15. Jeong SM, Choi BH, Kim J, Lee DH, Xuan F, Mo D et al. Comparison of flap and flapless proceduresfor stability of chemically modified SLA titanium implants: an experimental study in canine model. Oral Surg Oral Med Oral Pathol Oral Radiol Endod. 2011 ; 111 (2):170-173. doi: 10.1016/i. tripleo.2010.04.024

16. Kawai Y, Taylor J. Effect of loading time on the success of complete mandibular titanium implant retained overdentures: a systematic review. Clin Oral Implants Res. 2007;1 8(4):399408. doi: $10.1111 /$ i.1600-0501.2007.01376.x

17. Koutrach M, Nimmo A. Preservation of existing softtissue contours in the transition from a tooth to an implant restoration in the esthetic zone using a flapless approach: a clinical report. J Prosthodont. 2010;19(5):391-6. doi: $10.1111 /$ i.1532-849X.2010.00581.x

18. Lisboa MRP, Oliveira RN, Soratto A, Moreira MMSM. Biomodelos em implantodontia - relato de caso clínico. Rev Odontol UNESP. 2010;39(especial):117.

19. Malo P, Nobre MA, Lopes A. The use of computerguided flapless implant surgery and four implants placed in immediate function to support a fixed denture: preliminary results after a mean follow-up period of thirteen months. J Prosthet Dent. 2007; 97(Supl 6):S26-34. doi: 10.1016/ $\underline{\text { s0022-3913(07)60005-5 }}$

20. Menezes PDF, Sarmento V, Lamberti P. Aplicação da prototipagem rápida em implantodontia. Innovations Implant Journal - Biomaterials And Esthetics. 2008;3(6):39-44.

21. Moreschi E, Gottardo VD, Trento CL, Zamponi M, Zardetto Jr. R, Aleixo TRC. Cirurgia guiada por computador associada a função imediata: análise de um ano de acompanhamento clínico. Revista Implant News 2011 ; 8(1):20-4.

22. Pettersson A, Kero T, Gillot L, Cannas B, Fäldt J, Söderberg R, Näsström K. Precisão da cirurgia de implante de modelo cirúrgico guiado CAD / CAM em cadáveres humanos: Parte I. J Prosthet Dent. 2010;103(6):334-342.

23. Schramm A, Gellrich NC. Navegação intra-operatória e computador automotivo. In: Schwenzer N, Ehrenfeld $M$, editores. Zahn-Mund-Kiefer-Heilkunde / Mund-, Kiefer-, Gesichtschirurgie. Stuttgart, Nova lorque: Thieme; 2011 . pp. 478-499.

24. Schramm A, Wilde F. Die computergestützte Gesichtsschädelrekonstruktion. HNO. 2011 ; 59(8):800-806. doi: $10.1007 / \mathrm{s} 00106-011-2351-7$

25. Simon, H. Use of transitional implants to support a surgical guide: enhancing the accuracy of implant placement. J Prosthet Dent. 2002;87(2):229-232.
26. Sudbrink SD. Computer-guided implant placement with immediate provisionalization: a case report. J Oral Maxilosa Surg. 2005;63(6):771-4. doi: 10.1016/i.joms.2005.02.011

27. Behneke A, Burwinkel M, Knierim K, Behneke N. Accuracy assessment of cone beam computed tomography-derived laboratory-based surgical templates on partially edentulous patients. Clin Oral Implants Res. 2012;23(2):137-143. doi: 10.1111/i.1600-0501.2011.02176.x

28. Behneke A, Burwinkel $M$, Behneke N. Factors influencing transfer accuracy of cone beam CT-derived template-based implant placement. Clin Oral Implants Res. 2012;23(4):416423. doi: $10.1111 /$ i.1600-0501.2011.02337.x

29. Neugebauer J, Stachulla G, Ritter L, Dreiseidler T, Mischkowski RA, Keeve E et al. Computer-aided manufacturing technologies for guided implant placement. Expert Rev Med Devices. 2010;7(1):1 13-129. doi: 10.1586/ erd.09.61

30. Viegas VN. Avaliação das variações da transferência do planejamento virtual e sobre protótipos biomédicos para a instalação de implantes dentários pela técnica de cirurgia guiada [tese]. Porto Alegre: Pontifícia Universidade Católica do Rio Grande do Sul; 2008.

31. Widmann G, Bale RJ. Accuracy in computer-aided implant surgery--a review. Int J Oral Maxillofac Implants. 2006;21(2):305-313. 